\title{
Editorial
}

\section{Infarto agudo al miocardio sin lesiones coronarias significativas}

\author{
Alejandro Fajuri \\ División de Enfermedades Cardiovasculares, Pontificia Universidad Católica de Chile
}

El Infarto agudo al miocardio ocurre como consecuencia de una prolongada e irreversible isquemia miocárdica y presupone el cese total o subtotal de la perfusión al área infartada.

Como fuera señalado por Arnett y Roberts en 1976, no había demostraciones de permeabilidad del vaso coronario responsable durante la evolución de un infarto del miocardio, a pesar de algunos casos clínicos publicados en la década de los 60 y $70 .^{1}$

¿Cómo podríamos explicar el hallazgo de infartos sin lesiones coronarias sinificativas?

\section{Tres explicaciones son posibles:}

1. El infarto es el resultado de un espasmo coronario.

2. La coronariografía falló en detectar la obstrucción coronaria.

3. Se produjo una rápida recanalización o lisis del trombo responsable.

El interesante trabajo de Maldonado et al, presentado en este número de la Revista, ${ }^{2}$ es la primera publicación nacional a gran escala donde se destacan las características clínicas de pacientes con infarto reciente del miocardio sin lesiones coronarias significativas. Para ello se analizaron los datos del registro GEMI durante 2 años consecutivos incluyendo 3194 pacientes con infarto del miocardio a los que se les efectuó una coronariografía durante la hospitalización. De ellos, 116 (3.6\%) no tenían lesiones coronarias significativas. En una serie menor, Cortell et al encuentran un porcentaje mayor de pacientes sin lesiones significativas; sin embargo sólo incluyeron infartos no Q. ${ }^{4}$ La serie de Larsen et al, reporta un porcentaje aún más bajo que Maldonado et al, pero sólo incluyen pacientes con coronarias angiográficamente normales. ${ }^{4}$

Probablemente, estas diferencias se explican por el diagnóstico clínico (tipo de infarto) y por las características de las angiografías (coronarias angiográficamente normales vs coronarias con lesiones no significativas).

Por cierta lógica, la edad de estos pacientes es algo menor y los factores de riesgo coronario menos prevalentes. Estos hallazgos también se encuentran en publicaciones internacionales manifestándose en una aterosclerosis menos avanzada. . $^{3-4}$

Como también era esperable, estos infartos son de menor tamaño y por ende tienden a comprometer menos la función ventricular, confirmando la hipótesis del beneficio de "la arteria abierta"5. Este hecho, sin embargo, no se asoció

Correspondencia:

Dr. Alejandro Fajuri

División de Enfermedades Cardiovasculares, Pontificia Universidad Católica de Chile afajuri@med.puc.cl 
a una menor mortalidad intrahospitalaria en esa serie, pero es posible que si se manifieste en el largo plazo.

En el análisis multivariado aparece el sexo femenino como el principal predictor de infarto sin lesiones coronarias significativas. Este hallazgo se repite en series internacionales; sin embargo, esta información no nos debe inducir a equívocos. Ha sido demostrado en múltiples estudios que los infartos del miocardio en mujeres tienen mayor mortalidad y complicaciones que en el caso de los hombres, aún cuando existen controversias si el sexo es un predictor independiente de este exceso de mortalidad. ${ }^{6-7}$

De los datos presentados llama poderosamente la atención la mayor incidencia de angor crónico en los pacientes sin lesiones coronarias significativas. Tal como lo señalan los autores, no hay una explicación clara para este hallazgo. Quizás, a modo de especulación, podría plantearse la posibilidad de que corresponda a "angina microvascular" como expresión de enfermedad de la microcirculación.

De la terapia ofrecida a estos pacientes durante la hospitalización, destaca el menor uso de antiagregantes plaquetarios lo que, sin duda, se vió influenciado por los hallazgos angiográficos. Sin embargo, probablemente todos ellos debieran recibir antiagregantes ya que sabemos que ellos juegan un rol en la prevención de reoclusión coronaria ${ }^{8-9}$ y no podemos asegurar que en algún momento no hayan tenido una oclusión o suboclusión coronaria.

Los B Bloqueadores están universalmente recomendados, salvo contraindicaciones, como una terapia esencial en todos los síndromes coronarios agudos ${ }^{8-9}$, con la excepción de aquellos asociados al consumo reciente de cocaína y a la llamada "angina variante "o de Prinzmetal. El mero hecho de no encontrarse lesiones coronarias significativas per se no debiera ser un argumento para no utilizarlos, salvo que se haya documentado un fenómeno de espasmo coronario como factor causal del infarto.

Tampoco el subuso de estatinas parece razonable. En todos los infartos, independiente de los hallazgos angiográficos se deben utilizar, no olvidando los efectos pleiotrópicos de estos fármacos. ${ }^{8-9}$

Las pricipales indicaciones de los bloqueadores del calcio en el infarto se refieren al manejo de angina recurrente, por lo que su utilización es generalmente limitada. El mayor uso en estos casos, seguramente se fundamentó en la posibilidad de espasmo coronario como factor etiopatogénico. Por último vale la pena señalar que el buen pronóstico de los infartos no asociados a lesiones coronarias significativas no significa no indicar todas las medidas de prevención secundaria recomendadas independientes de los hallazgos angiográficos. 
1. ARNETT EN, ROBERTS WC. Acute myocardial infarction and angiographically normal coronary arteries: An unproven combination. Circulation 1976; 53: 395.

2. MALDONADO R, MADARIAGA A , LÓPEZ C, NAZZAL C , PRIETO JC. Caracterización clínica de pacientes con infarto agudo al miocardio sin lesiones coronarias significa tivas .GEMI 2011-2013. Rev Chil Cardiol 2014; 33: 165-172.

3. CORTELL A, SANCHISA J, BODÍA V, NÚÑEZ J, MAINARA L, PELLICER M, et al. Infarto de miocardio sin elevación del ST con coronarias normales: predictores y pronóstico. Rev Esp Cardiol. 2009; 62:1260-6.

4. LARSEN AI, GALBRAITH PD, GHALI WA, NORRIS CM, GRAHAM MM, KNUDTSON ML, et al. Characteristics and outcomes of patients with acute myocardial infarction and angiographically normal coronary arteries. Am J Cardiol 2005; 95:261-3

5. WHITE HD, CROSS DB, ELLIOTT JM, NORRIS RM, YEE
TW. Long-term prognostic importance of patency of the infarct-related coronary artery after thrombolytic therapy for acute myocardial infarction. Circulation.1994; 89: 61-67.

6. VACCARINO V, KRUMHOLZ HM, BERKMAN LF, HORWITZ RI. Sex differences in mortality after myocardial infarction: is there evidence for an increased risk for women? Circulation 1995;91:1861-71.

7. MALACRIDA R1, GENONI M, MAGGIONI AP, SPATARO V, PARISH S, PALMER A, et al. A Comparison of the Early Outcome of Acute Myocardial Infarction in Women and Men. N Engl J Med 1998; 338:8-14

8. $2013 \mathrm{ACCF} / \mathrm{AHA}$ Guideline for the Management of ST-Elevation Myocardial Infarction. JACC 2013 Vol. 61, No. 4.

9. 2014 AHA/ACC Guideline for the Management of Patients With Non-ST-Elevation Acute Coronary Syndromes 2014, VOL. 64, NO. 24. 